\title{
Ökonomische Chancen der deutschen Industrie resultierend aus einer weltweiten Verbreitung von CSP (Concentrated Solar Power)- Technologien
}

Originally published as:

Daniel Vallentin, Peter Viebahn (2011):

Ökonomische Chancen der deutschen Industrie resultierend aus einer weltweiten Verbreitung von CSP (Concentrated Solar Power)-Technologien In: Zeitschrift für Energiewirtschaft 35(1) 53-60

DOI: 10.1007/s12398-010-0034-9 
Daniel Vallentin ${ }^{a,{ }^{*}}$, Peter Viebahn ${ }^{a}$

Ökonomische Chancen der deutschen Industrie resultierend aus einer weltweiten Verbreitung von CSP (Concentrated Solar Power)Technologien

a Wuppertal Institute for Climate, Environment and Energy, Germany

* Corresponding author: Daniel Vallentin, Wuppertal Institute, Neue Promenade 6, 10178 Berlin, Germany

E-mail: daniel.vallentin@wupperinst.org

Phone: +49-030-2887458-12

Fax: $\quad+49-030-2887458-40$ 


\section{Einleitung}

Um das vom Weltklimarat (IPCC) geforderte $2^{\circ} \mathrm{C}$-Ziel einhalten zu können, ist eine Reduktion der globalen $\mathrm{CO}_{2}$-Emissionen um $80 \%$ bis 2050 gegenüber dem Stand von 1990 zwingend notwendig. Hierbei wird auch solarthermischen Kraftwerken eine immer größere Bedeutung beigemessen. Im BLUE Map-Szenariio der Internationalen Energieagentur (IEA), das von einer $\mathrm{CO}_{2}$-Reduktion um $50 \%$ bis 2050 gegenüber 2005 ausgeht, müssen im Jahr 2050 ca. 11\% (4.754 TWh) des weltweiten Strombedarfs durch Sonnenenergie gedeckt werden (IEA 2008). Neben Photovoltaik sollen solarthermische Kraftwerke (Concentrated Solar Power, CSP) etwa 46\% (ca. 2.200 TWh) der prognostizierten Menge an Solarstrom erzeugen. Im Energy[R]evolution Szenario von Greenpeace International und EREC (European Renewable Energy Council) aus dem Jahr 2008 werden rund 6.000 TWh an CSP-Strom im Jahr 2050 angenommen (bei einer installierten Leistung von $801 \mathrm{GW}$ ) (Greenpeace International et al. 2008), während andere Studien bis zu $1.000 \mathrm{GW}$ installierter Leistung in 2050 betrachten (Viebahn et al. 2010). Die DESERTEC-Initiative gibt ein Ziel von $5.000 \mathrm{GW}$ installierter Leistung im Jahr 2050 vor (Knies 2009).

Der Export von CSP-Technologien in die „Sunbelt“-Regionen bietet große Chancen für deutsche Anlagenbauer. So sind u.a. Schott Solar, die Ferrostaal Group mit ihrem Geschäftssegment "Solar Energy“, Flagsol, die Solar Power Group, Solar Millenium und Fichtner Solar auf dem Gebiet CSP aktiv. Schott Solar (Receiver) und Flabeg (Spiegel) haben eine weltweit führende Markstellung inne. Große deutsche Energieversorger und Anlagenbauer wie E.On, RWE und Siemens gehören zum Industriekonsortium der Desertec Industrial Initiative, die den Ausbau von CSP in der MENA-Region vorantreiben will. Die Initiative wurde von der Münchener Rück angestoßen.

In diesem Artikel wird dargestellt, welche Aktivitäten deutsche Unternehmen entlang der Wertschöpfungskette bislang aufweisen und wie ihre Marktstellung im Vergleich zu führenden internationalen Unternehmen zu bewerten ist. Anschließend wird auf Basis von vorliegenden Energieszenarien ermittelt, welche messbaren ökonomischen Effekte für deutsche Unternehmen, z.B. zusätzliche Wertschöpfung und die Schaffung neuer Arbeitsplätze, aus den genannten Potentialen resultieren. Die Ergebnisse basieren auf einer Studie des Wuppertal Instituts, die im Auftrag von Greenpeace Deutschland und der DESERTEC Foundation erstellt wurde (Vallentin und Viebahn 2009).

\section{CSP-Technologiepfade}

Derzeit konzentriert sich die internationale CSP-Debatte auf zwei Technologieoptionen: Parabolrinnenanlagen sowie Solarturmanlagen. Bei anderen Optionen, wie Dish Stirling-Motoren oder Fresnel-Systeme, bestehen bislang nur sehr begrenzte Erfahrungen mit kommerziellen Anlagen. Sie stehen daher nicht im Mittelpunkt der Debatte.

Parabolrinnensysteme sind die am besten erprobte und derzeit wirtschaftlichste Technologie zur solarthermischen Stromerzeugung. Zunächst wird die Strahlung durch parabolförmige, einachsige Kollektoren gebündelt. In der Brennlinie des Spiegels befindet sich ein Absorberrohr, durch welches ein Arbeitsmedium fließt, das aufgrund der stark konzentrierten Strah-lung auf bis zu $400^{\circ} \mathrm{C}$ erhitzt wird. Derzeit werden für diesen Zweck synthetische Thermo-Öle oder Salzschmelzen verwendet. Seit den 1980er Jahren wird die Parabolrinnentechnologie in Kalifornien in neun "Solar Electricity Generating Systems“ (SEGS) mit einer Erzeugungskapazität von insgesamt $354 \mathrm{MW}_{\text {el }}$ kommerziell genutzt. Weltweit sind inzwischen Parabolrinnenkraftwerke mit einer Kapazität von mehr als $850 \mathrm{MW}_{\text {el }}$ in Betrieb, mehr als $1.800 \mathrm{MW}_{\mathrm{el}}$ sind im Bau. Der durchschnittliche Jahreswirkungsgrad von Parabolrinnenkraftwerken liegt bei 9-14\% (Viebahn et al. 2010). Der Wirkungsgrad der Anlagen wird insbesondere durch die begrenzte Temperatur des Arbeitsmediums limitiert, weshalb derzeit im Rahmen von FuE-Projekten die Alternative entwickelt wird, Direktdampf anstatt Thermoöl zu verwenden (Direct Steam Generation, DSG). Spanien ist aufgrund eines speziellen Einspeisetarifs ein Schlüsselmarkt für solarthermische Kraftwerke. Als „Vorreiterprojekte“ gelten die spanischen $50 \mathrm{MW}_{\mathrm{el}}$-AndasolKraftwerke I und II, die im Jahre 2008 bzw. 2009 fertig gestellt wurden. Die Nachfolgeprojekte Andasol III und IV mit einer Kapazität von jeweils $50 \mathrm{MW}_{\text {el }}$ sowie zahlreiche weitere Anlagen befinden sich aktuell im Bau. In Algerien, Marokko und Ägypten sind Hybrid-Anlagen, die die 
Parabolrinnentechnologie mit einem Erdgas gefeuerten Gas- und Dampfprozess kombinieren, mit einer Gesamtkapazität von $60 \mathrm{MW}_{\mathrm{el}}$ im Bau. Torresol errichtet zwei Parabolrinnenanlagen (Valle 1 und Valle 2) mit einer Kapazität von jeweils $50 \mathrm{MW}_{\mathrm{el}}$ in Andalusien.

In Solarturmkraftwerken wird die Sonnenstrahlung durch ein großes Feld einzeln nachgeführter, nahezu flacher Planspiegel (Heliostaten) auf einen Turm fokussiert. Ein auf dem Turm angebrachter Strahlungsempfänger absorbiert die Strahlungsenergie und gibt die Energie an ein Wärmeträgermedium ab. Die Temperatur des Mediums liegt meist zwischen $500-800{ }^{\circ} \mathrm{C}$. Als Wärmeträger können direkt erzeugter Dampf, Salzschmelzen oder Luft verwendet werden. Solarturmsysteme haben den Vorteil, dass die Energieübertragung ledig-lich an einem Punkt des Verfahrens erfolgt und dadurch der Energiebedarf für den Energie-transport reduziert wird. Der Gesamtwirkungsgrad von Solarturmsystemen beträgt 13-18\% (Viebahn et al. 2010). Durch eine Erhöhung der Temperatur des Wärmeträgermediums könnte der Wirkungsgrad um $20 \%$ erhöht werden (ESTELA 2009).

Ein Vorteil von Turmkraftwerken ist, dass diese Technologie durch die hohen Temperaturen am Receiver für den Betrieb von Gas- und Dampfturbinen geeignet ist. In einer Pilotanlage in Almeria konnte das Deutsche Zentrum für Luft- und Raumfahrt (DLR) bis zu $1.000{ }^{\circ} \mathrm{C}$ heiße Luft erzeugen und damit eine Gasturbine antreiben. Diese Technologie bietet sich daher auch für Hybridanlagen mit hohem Gesamtwirkungsgrad an (Zufeuerung durch Biomasse oder Erdgas in den Zeiten, in denen die Sonne nicht scheint oder die Temperatur im Auslegungspunkt nicht erreicht wird).

Gegenwärtig sind weltweit nur wenige Solarturmkraftwerke in Betrieb. In der spanischen Provinz Sevilla befindet sich das erste "neuere“ PS10-Kraftwerk von Abengoa, einem spani-schen Anlagenbauer. Es hat eine Kapazität von $10 \mathrm{MW}_{\text {el }}$, nutzt Dampf als Wärmeträgerme-dium und ist mit einem thermischen Energiespeicher $\left(20 \mathrm{MWh}_{\text {th }}\right.$ ) ausgestattet. Die Anlage ist seit Februar 2007 in Betrieb. Am gleichen Standort hat ein weiteres Solarturmkraftwerk (PS 20) mit einer Kapazität von $20 \mathrm{MW}_{\text {el }}$ Ende April 2009 den Betrieb aufgenommen. Torresol errichtet derzeit ebenfalls in der Provinz Sevilla eine $17 \mathrm{MW}_{\mathrm{el}}$-Anlage. Torresol ist zu 60\% im Besitz von Masdar, der Initiative für Erneuerbare Energie in Abu Dhabi, und zu 40\% in den Händen von Sener, einer spanischen Ingenieursfirma. Die Gesamtkapazität weltweit angekündigter Solarturmprojekte liegt bei mehr als $1.500 \mathrm{MW}_{\mathrm{el}}$.

In Deutschland wurde im Jahr 2009 der Testbetrieb eines 1,5 $\mathrm{MW}_{\mathrm{el}}$-Solarturmkraftwerks in Jülich erfolgreich abgeschlossen. Das Projekt wurde von den Stadtwerken Jülich in Auftrag gegeben und wird federführend von Kraftanlagen München (KAM) durchgeführt. Hauptziel sind hier die Entwicklung und perspektivisch der Export der verwendeten Technologie.

\section{Aktivitäten und Marktstellung deutscher Unternehmen entlang der Wertschöpfungsket- te}

Deutsche Unternehmen haben bei der Konzeption, der Planung und dem Bau von CSPKraftwerken weltweit eine prominente Stellung inne. Nachfolgend werden die wichtigsten deutschen Marktspieler entlang der CSP-Wertschöpfungskette kurz dargestellt.

Engineering and Services: Zu den prominentesten deutschen Marktakteuren auf diesem Gebiet gehören Schlaich Bergermann und Partner (SBP) sowie Fichtner Solar. Beide Unternehmen sind etablierte Martkspieler mit einem hohen Maß an Umsetzungserfahrung und Referenzprojekten. SBW war an der Entwicklung und Planung von Parabolrinnenanlagen in Spanien (Andasol I-III), Ägypten und den USA beteiligt. Fichtner Solar ist seit mehr als 25 Jahren auf dem Gebiet der CSP-Technologien aktiv und verfügt über ein weltweites Netzwerk an Tochterunternehmen, die bei der Umsetzung von Projekten über Kenntnisse der jeweiligen örtlichen Begebenheiten verfügen. Fichtner Solar war bislang in CSP-Projekte mit einem finanziellen Gesamtvolumen von $5 \mathrm{Mrd}$. $€$ beteiligt. Hierzu gehören u.a. Hybrid-Anlagen in Ägypten, Indien und Marokko. Als weitere wichtige deutsche Marktakteure sind CSP Services, Lahmeyer International und Flagsol zu nennen. Die Flagsol GmbH ist seit 2005 eine $100 \%$ ige Tochter der Solar Millennium AG. CSP Services ist eine Ausgründung des Deutschen Zentrums für Luft- und Raumfahrt zur Vermarkt von dessen Know-how, Equipment und Analysetools für die Qualifizierung der Spiegelfertigung. Im internationalen Vergleich sind deutsche 
Engineering and Services-Unternehmen gut positioniert, befinden sich jedoch insbesondere mit spanischen und U.S.-amerikanischen Unternehmen in starkem Wettbewerb.

Komponentenzulieferer: Deutsche Komponentenzulieferer wie Schott Solar, Flabeg oder Siemens zählen zu den weltweit führenden CSP-Technologienanbietern und verfügen im internationalen Vergleich über herausragendes, hochgradig spezialisiertes Know-how. Die Herstellung hochgradig spezialisierter und technisch komplexer Anlagenkomponenten ist die Stufe der CSP-Wertschöpfungskette, auf der deutsche Unternehmen vergleichsweise am stärksten positioniert sind. Laut Kistner (2009) könnte die deutsche Beteiligung an Wertschöpfungseffekten resultierend aus einem Ausbau von CSP deutlich erhöht werden, wenn die deutsche Industrie ihre Expertise auf strategisch relevanten Gebieten, wie die Produktion von Turbinen oder Solarfeldern, ausbauen würde. Die Aktivitäten deutscher Industrieunternehmen umfassen nahezu alle Prozessstufen der CSP-Verfahren. Schott Solar ist beispielsweise der weltweit führende Hersteller von Receivern für Parabolrinnenanlagen. Neben anderen Anlagen wird Schotts Technologie im Andasol I-Kraftwerk eingesetzt. Das Unternehmen ist auf allen CSPSchlüsselmärkten, wie den USA, Südeuropa oder dem Nahen Osten, aktiv.

Die Flabeg Gruppe produziert hoch präzise Solar Reflectors für solarthermische Kraftwerke. Überdies leistet das Unternehmen die Befestigung der Panels und verfügt über Systeme, um deren Qualität zu kontrollieren. Beginnend mit den in den 1980er Jahren errichteten SEGSKraftwerken in den USA, hat Flabeg für praktisch alle existierenden und im Bau befindlichen Parabolrinnenkraftwerke die Spiegel geliefert. Dies summiert sich zu einer Gesamtkapazität von 1,3 GW (Flabeg 2009).

Andere wichtige deutsche Komponentenzulieferer sind Senior Berghöfer, Züblin und Siemens. Die Erneuerbare Energien-Sparte von Siemens produziert speziell für solarthermische Kraftwerke ausgelegte Dampfturbinen, die in Anlagen mit Kapazitäten von bis zu $175 \mathrm{MW}_{\mathrm{el}}$ operieren können. Bis zum Jahr 2020 erwartet Siemens ein Wachstum des CSP-Marktes um 20\%. Die hierfür notwendigen Kapazitäten versucht Siemens durch die Übernahme etablierter CSPUnternehmen, wie z.B. das israelische Unternehmen Solel Solar Systems im Oktober 2009, zu akquirieren.

Realisierung von CSP-Kraftwerken: Zu deutschen Generalunternehmern, welche die Gesamtrealisierung von CSP-Anlagen verantworten, zählen Ferrostaal, Solar Millennium, Kraftanlagen München und Novatec Biosol. Solar Millennium stellt dabei den wichtigsten Marktspieler dar. Aktuell wird der internationale Markt jedoch von spanischen Unternehmen, z.B. Abengoa Solar, Solel Aries oder Iberdrola, dominiert. In den kommenden Jahren wird sich der Wettbewerb voraussichtlich noch verschärfen, da zunehmend Unternehmen aus dem konventionellen Energieerzeugungssektor auf den CSP-Markt drängen.

Solar Millennium war u.a. an Andasol I und II beteiligt. Es ist auf allen Stufen der CSPWertschöpfungskette aktiv und hat eine 50:50-Beteiligung mit Ferrostaal gegründet. Kraftanlagen München und Novatec Biosol sind eher kleine Marktteilnehmer mit einer begrenzten Zahl an Referenzprojekten. KAM ist u.a. Generalunternehmer des Solarturmkraftwerks in Jülich.

\section{Szenarienmethodik}

Nachfolgend wird der Anteil deutscher Unternehmen an der Wertschöpfungskette resultierend aus einem Ausbau der CSP-Technologie ermittelt. Dabei werden Zahlen existierender Studien als Basis genutzt. Konkret werden drei Szenarien miteinander verküpft, die für die Berechnung des deutschen Anteils an der weltweiten Wertschöpfung durch CSP verwendet werden:

Technologieentwicklungsszenarien: Greenpeace International und Solarfachverbände haben im Mai 2009 eine Studie vorgelegt, die Szenarien für den weltweiten Ausbau der CSPTechnologie bis zum Jahr 2050 entwirft (Greenpeace International et al. 2009). Diese Technologieentwicklungsszenarien für CSP fungieren in der folgenden Wertschöpfungsanalyse als Grundlage für die Darstellung möglicher ökonomischer Chancen der deutschen Industrie durch einen globalen Ausbau von CSP. Die Studie umfasst einen moderaten und einen ehrgeizigen CSP-Ausbaupfad. Ersterer basiert auf den weltweit existierenden und geplanten Maßnahmen zum Ausbau erneuerbarer Energien. Letzerer untersucht, in welchem Maße die CSP-Industrie im bestmöglichen Fall wachsen könnte. 


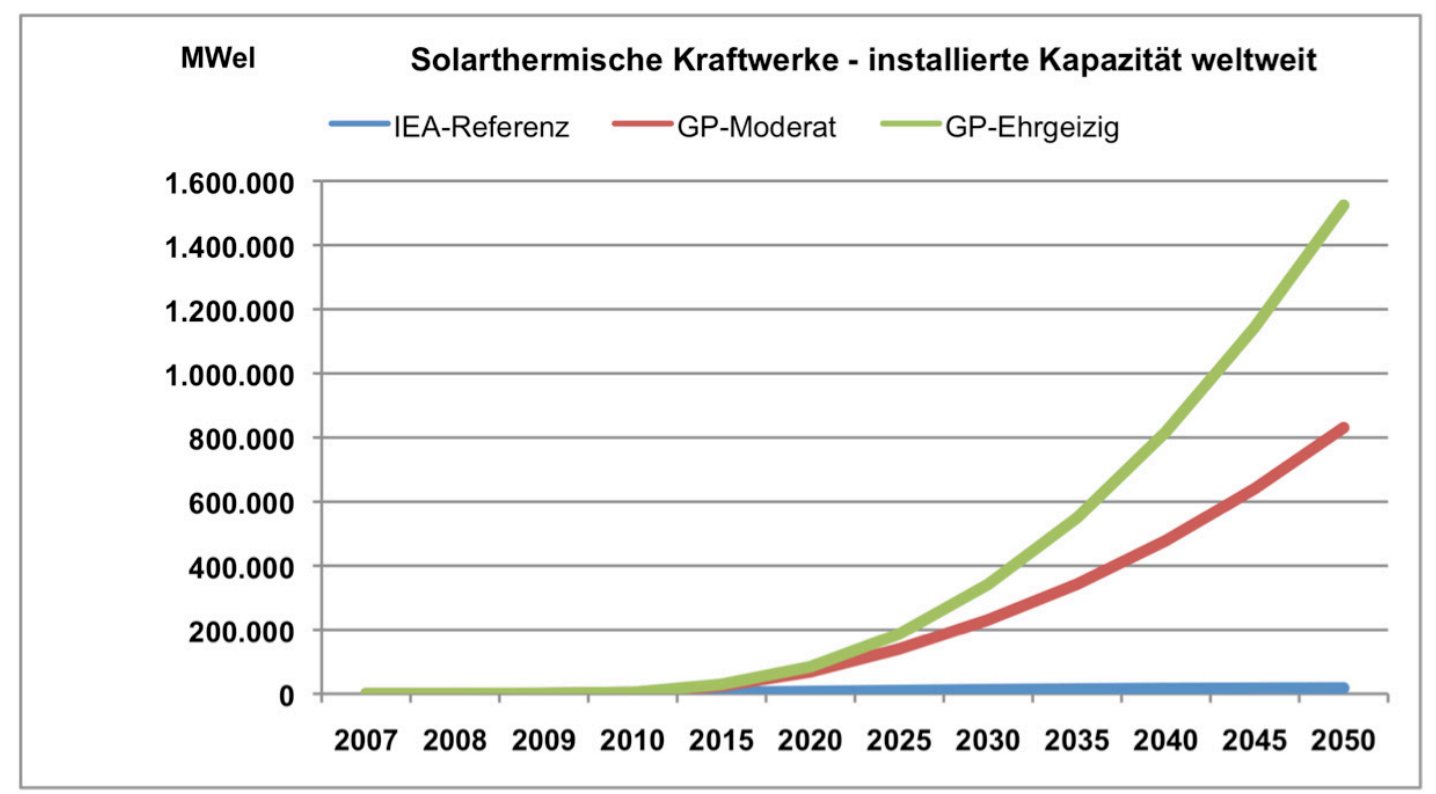

Abb. 1 Greenpeace-Szenarien zum weltweiten Ausbau von CSP-Kraftwerken

Quelle: Greenpeace International et al. 2009

Dem ehrgeizigen Szenario liegt die Annahme zugrunde, dass alle Politikempfehlungen industrieller Akteure für erneuerbare Energien weltweit erfolgreich umgesetzt werden. Des weiteren wird von einer starken und koordinierten Zunahme der Netzkapazitäten zur Übertragung des Stroms ausgegangen. Beide Szenarien werden durch ein konservatives Referenzszenario der Internationalen Energieagentur (IEA) ergänzt. Dieses berücksichtigt lediglich existierende Maßnahmen zum Ausbau erneuerbarer Energien, legt jedoch keine weiterführenden Maßnahmen zugrunde. Abb. 1 zeigt den von den dargestellten Szenarienvarianten angenommenen weltweiten Ausbau von CSP bis zum Jahr 2050.

Kostenszenarien: Zur Ermittlung der jährlichen Wertschöpfung aus dem Zubau von Solarkraftwerken werden die jährlichen Investitionskosten pro Kraftwerk benötigt. Aus innen können im nächsten Schritt dann die weltweit zu tätigenden Investitionen berechnet sowie der Anteil der deutschen Industrie ermittelt werden. Zur Ableitung der spezifischen Investitionskosten wird im Folgenden der Lernkurvenansatz verwendet. Die Lernrate einer Technologie gibt an, um wieviel Prozent die Investitionskosten sinken, wenn sich die weltweit installierte Kapazität verdoppelt. Eine Lernrate von $15 \%$ bedeutet beispielsweise, dass pro Verdopplung der installierten Kapazität die spezifischen Kosten um 15\% sinken. Für die folgende Rechnung werden die innerhalb des EU-Projektes NEEDS für solarthermische Kraftwerke hergeleiten Lernraten verwendet (Viebahn et al. 2010). Aufgrund des unterschiedlichen Reifegrades der solarthermischen Technik wurden dort verschiedene Lernraten angewendet: Während der konventionelle Kraftwerksteil (Turbine, Balance of Plant,...) weitgehend ausgereift ist und hier nur die Anpassung an die spezifischen Gege-benheiten eines Solarkraftwerks erfolgen muss, wird für den Solarteil und den Speicher noch ein erhebliches Kostensenkungspotenzial angenommen. Als Anfangswert für das Jahr 2009 werden spezifische Investitionskosten von insgesamt 6.000 $€ / \mathrm{kW}_{\mathrm{el}}$ angesetzt. Abb. 2 zeigt die Entwicklung der CSP-Investitionskosten.

Wertschöpfungsszenarien: Der deutsche Anteil an der durch CSP-Investitionen generierten Wertschöpfung wird auf Basis einer Studie von Kistner (2009) ermittelt. Hier werden deutsche Wertschöpfungsanteile an einer als Referenzanlage untersuchten Parabolrinnenanlage ermittelt. Die Referenzanlage orientiert sich großteils an Kostendaten des Andasol III-Kraftwerks, das ebenfalls für eine Kapazität von $50 \mathrm{MW}_{\text {el }}$ ausgelegt wird. Es wird ebenso wie die anderen beiden Andasol-Kraftwerke mit einem thermischen Energiespeicher mit einer Speicherdauer von 7,5 Stunden (und damit einer Speicherkapazität von $375 \mathrm{kWh}_{\mathrm{el}}$ ) ausgestattet. Die Investitionskosten der Anlage belaufen sich auf 300 Millionen $€$ bzw. $6.000 € / \mathrm{kW}_{\mathrm{el}}$. 


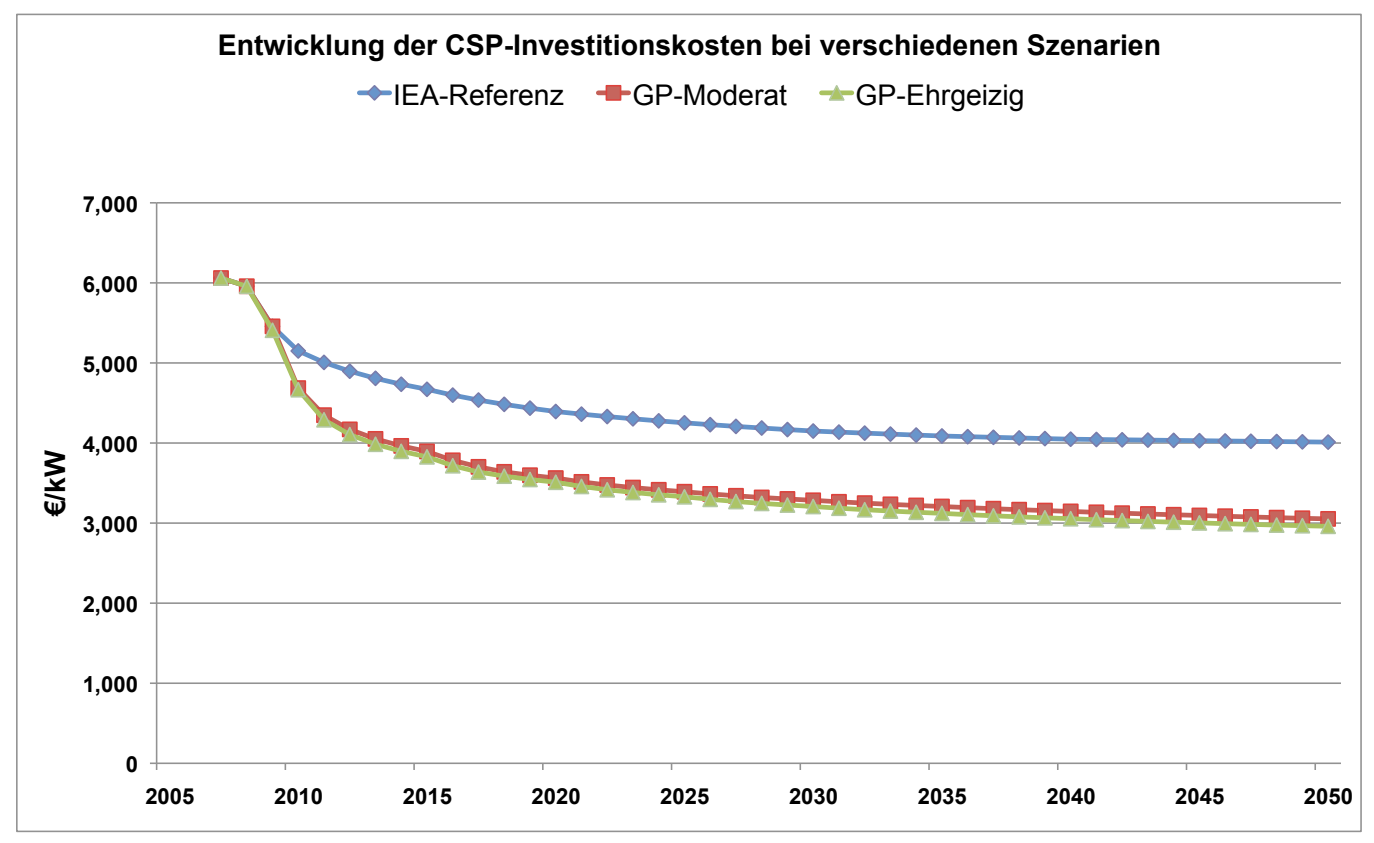

Abb. 2 Vergleich der Entwicklung der CSP-Investitionskosten für die drei Szenarienpfade Quelle: Eigene Darstellung

Basierend auf den aktuellen Produktionskapazitäten und der bestehenden Expertise geht Kistner (2009) davon aus, das deutsche Unternehmen ca. 33\% der EPC-Kosten (Engineering, Procurement, Construction) an der Referenzanlage generieren könnten. Wenn sich die Rahmenbedingungen für die Entwicklung und Kommerzialisierung von CSP verschlechtern, könnte dieser Anteil auf 10\% sinken. Dieser Fall könnte beispielsweise bei einem Verlust an Knowhow und Expertise oder einer zunehmenden Standardisierung der CSP-Komponenten eintreten. Wenn es deutschen Unternehmen hingegen gelänge, ihre Kompetenzen und Expertise auf strategisch wichtigen und umsatzstarken Gebieten auszubauen, geht Kistner davon aus, dass die deutsche Teilhabe an der CSP-Wertschöpfung auf ca. $41 \%$ der Referenzanlage ansteigt. Diese Annahmen sind, wie erwähnt, explizit auf die Parabolrinnentechnologie bezogen. Bei der Solarturmtechnologie müsste hingegen von geringeren deutschen Wertschöpfungsanteilen ausgegangen werden, da hier insbesondere U.S.-amerikanische Technologieanbieter wie BrightSource international führend sind.

Wenn deutsche Unternehmen mit 33\% an der Wertschöpfung partizipieren, fließen 33\% der generierten Umsätze an deutsche Marktakteure. Als deutsche Marktakteure werden hier Unternehmen eingestuft, deren Firmenzentralen sich in Deutschland befinden. Es war im Rahmen dieser Studie nicht möglich zu differenzieren, ob deutsche Unternehmen die Komponenten von CSP-Anlagen in Deutschland oder internationalen Fertigungsstätten produzieren lassen. Überdies muss darauf hingewiesen werden, dass die Studie von Kistner (2009) unter dem Dach der Ferrostaal Group erstellt wurde und die getroffenen Annahmen für potenzielle deutsche Wertschöpfungsanteile möglicherweise nicht völlig unabhängig sind. Mangels öffentlicher Verfügbarkeit unabhängiger Daten musste dennoch auf diese Studie zurückgegriffen werden.

\section{Wertschöpfung deutscher Unternehmen durch einen Ausbau von CSP}

Nachfolgend wird eine grobe Abschätzung der Wertschöpfung deutscher Unternehmen resultierend aus der globalen Verbreitung von CSP-Technologien basierend auf den beschriebenen Szenarien (Technologieentwicklungsszenarien, Kostenszenarien und Wertschöpfungsszenarien) dargestellt. Die Berechnung der deutschen Wertschöpfungschancen beruht auf vier Varianten: drei Varianten berechnen die von Kistner (2009) angenommenen Wertschöpfungsanteile deutscher Unternehmen an der Referenzanlagen (10\%, 33\%, 41\%) auf die einzelnen CSPSchlüsselkomponenten. Allerdings berücksichtigt Kistners Analyse nicht alle Kostenfaktoren des Referenzkraftwerks; wichtige Kostenfaktoren (z.B. Balance of Plant) bleiben außer Acht. Bei der vierten Variante werden daher die von Kistner im 33\%-Szenario angenommenen 
Wertschöpfungsanteile und Basisannahmen auf alle Kostenbestandteile des Referenzkraftwerkes angerechnet. Daraus resultiert ein deutscher Wertschöpfungsanteil von 37\%.

Abb. 3 illustriert die Beteiligung deutscher Unternehmen an der CSP-Wertschöpfung basierend auf dem konservativen IEA-Referenzszenario. Dieses weist zwar einen Zubau an CSPKapazitäten aus, jedoch geht die Menge an jährlich zugebauter Kraftwerksleistung und der damit einhergehenden Investitionen ab 2020 stetig zurück. Dementsprechend steigen auch die Umsatzanteile deutscher Unternehmen in allen vier Varianten zunächst an und nehmen ab dem Jahr 2020 kontinuierlich wieder ab. Im Maximalfall (2016-2020) können sie Umsätze zwischen 1,4 und 5,9 Mrd. € pro Jahrfünft erreichen, im Minimalfall (2046-50) nur noch zwischen 0,3 und 1,3 Mrd. $€$ pro Jahrfünft.

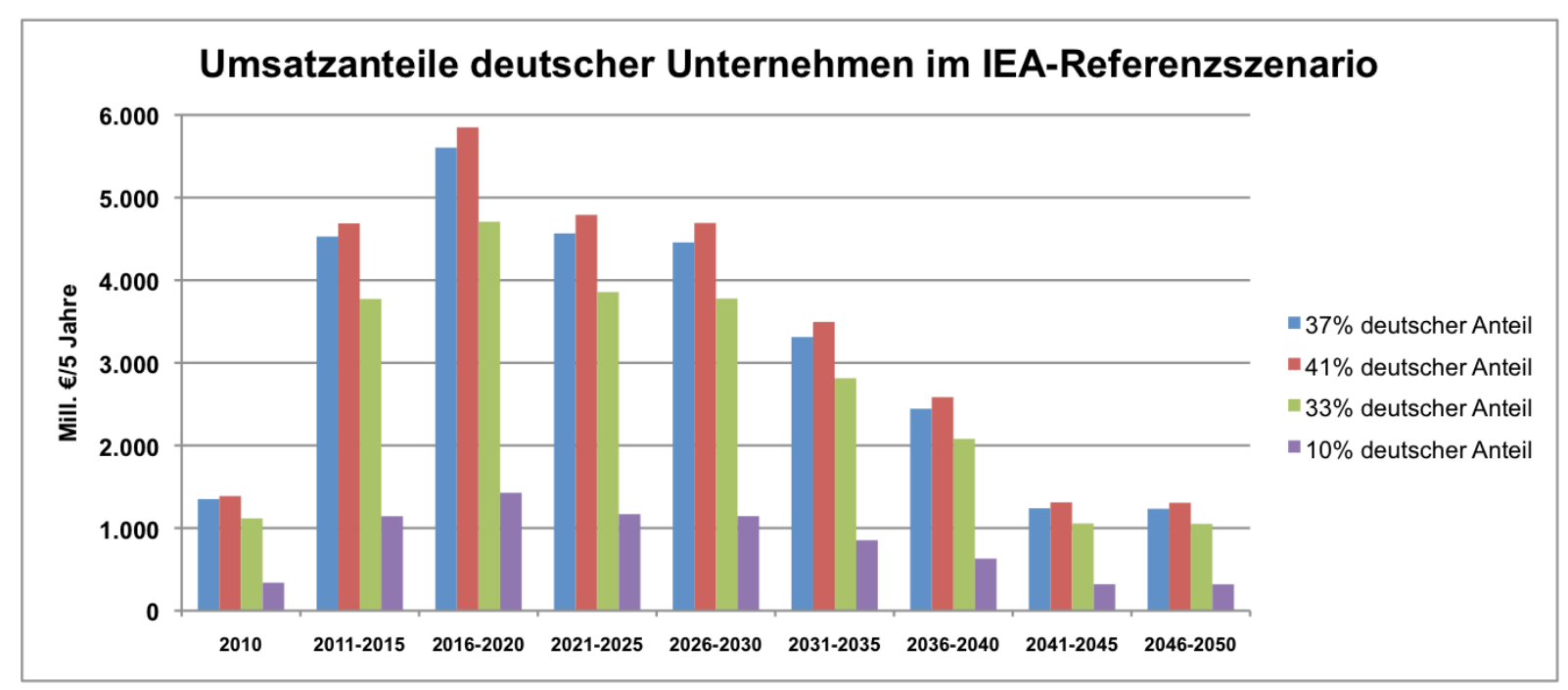

Abb. 3 Umsatzanteile deutscher Unternehmen an der Wertschöpfung durch einen weltweiten CSP Ausbau im IEA-Referenzszenario

Quelle: Eigene Darstellung

Aufgrund der deutlich stärkeren Ausbaudynamik im moderaten (Abb. 4) und ehrgeizigen Ausbaupfad (Abb. 5) fallen die Umsätze deutscher Unternehmen dort deutlich höher aus und steigen bis 2050 kontinuierlich an. Im moderaten Szenario startet die Entwicklung im Zeitraum von 2011-2015 mit Umsätzen zwischen 8,4 und 34,4 Mrd. € pro Jahrfünft und erreicht am Ende des Szenariozeitraums (2046-50) zwischen 58,3 und 239,1 Mrd. € pro Jahrfünft. Im ehrgeizigen Szenario betragen die Umsätze am Beginn zwischen 10,2 und 41,7 Mrd. € pro Jahrfünft und am Ende zwischen 113,1 und 463,7 Mrd. € pro Jahrfünft.

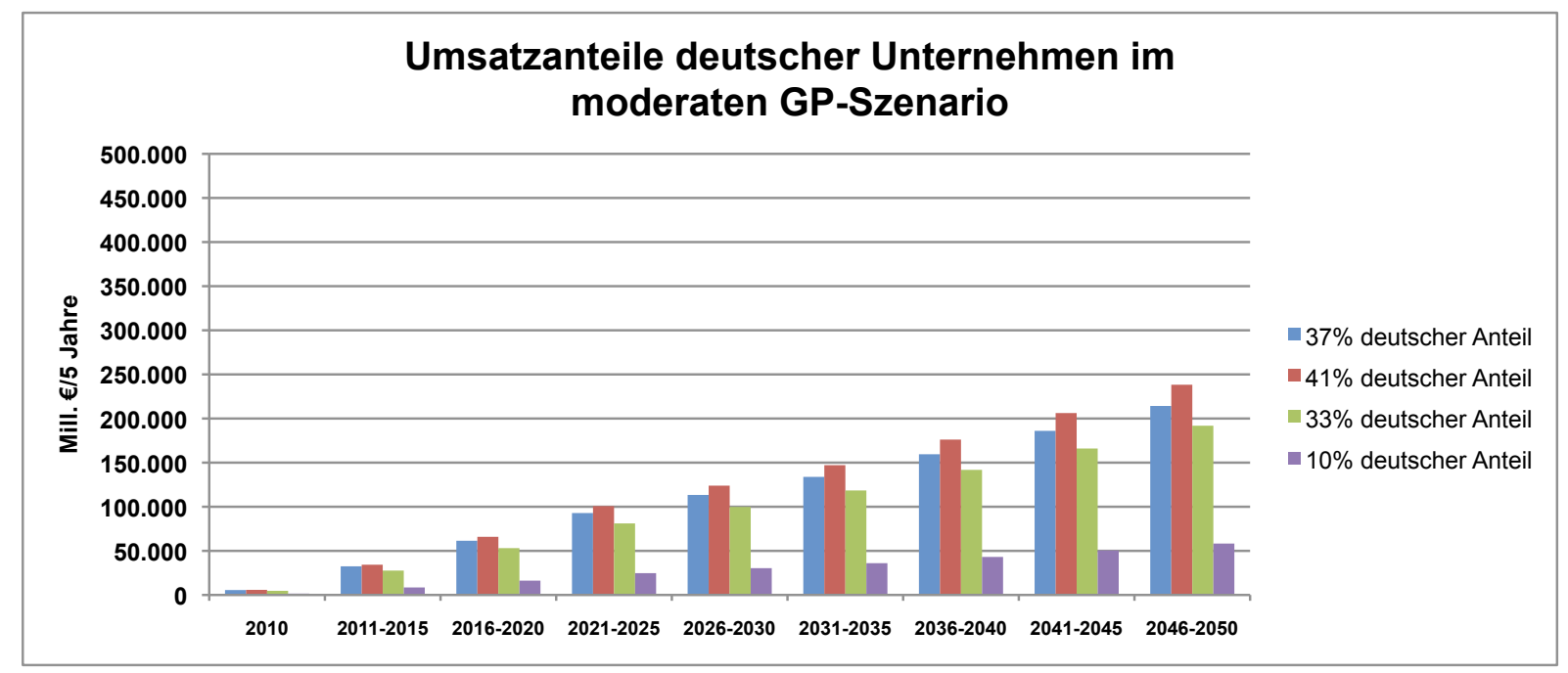

Abb. 4 Umsatzanteile deutscher Unternehmen an der Wertschöpfung durch einen weltweiten CSP Ausbau im moderaten GP-Szenario

Quelle: Eigene Darstellung 


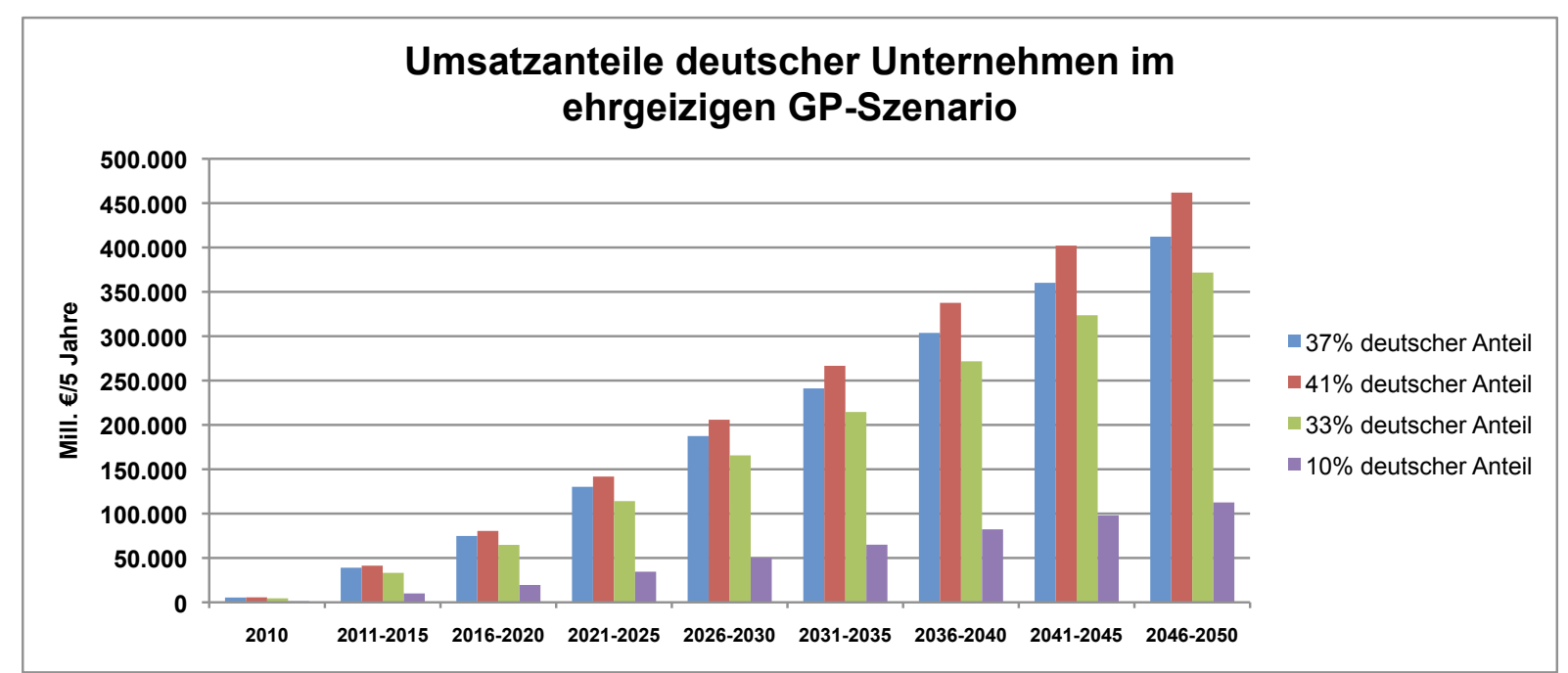

Abb. 5 Umsatzanteile deutscher Unternehmen an der Wertschöpfung durch einen weltweiten CSP Ausbau im ehrgeizigen GP-Szenario

Quelle: Eigene Darstellung

Das kumulierte Wertschöpfungspotential deutscher Unternehmen im Zeitraum von 2010 und 2050 summiert sich im Referenzpfad auf 7-30 Mrd. $€$. In den moderaten und ehrgeizigen Szenarien liegen die Wertschöpfungsanteile hingegen bei 269-1.102 Mrd. € bzw. 476-1.952 Mrd. $€$.

Die genannten Zahlen mögen sehr hoch erscheinen, allerdings stellen sie nur einen vergleichsweise geringen Teil der weltweit insgesamt erforderlichen Investitionen im Kraftwerkssektor in den kommenden Jahrzehnten dar. Die IEA geht davon aus, dass hier bei einer Fortschreibung der aktuellen Entwicklung zwischen 2005 und 2050 Investitionen in Höhe von etwa 12,7 Billionen $€$ (IEA 2008) notwendig sind.

\section{Schlussfolgerungen und Ausblick}

Es können die folgenden zentralen Schlussfolgerungen gezogen werden:

Deutschlands CSP-Industrie ist international gut positioniert. Dies gilt insbesondere für hoch spezialisierte Komponentenzulieferer wie Schott Solar oder Flabeg. Der Ausbau hoch spezialisierten Know-hows mit Blick auf strategisch wichtige und umsatzstarke Komponenten impliziert daher das größte zukünftige Wertschöpfungspotential für deutsche Technolo-gieanbieter.

Um die prognostizierte Nachfrage nach CSP-Technologien befriedigen zu können, müssten deutsche Marktakteure jedoch ihre Produktionskapazitäten deutlich ausbauen. Beispielsweise hat Siemens im Jahr 2009 seine Fertigungskapazitäten für Turbinenblöcke für solarthermische Kraftwerke ausgeweitet. Derzeit sind die Produktionskapazitäten der meisten CSPTechnologieanbieter noch begrenzt. Investitionen in die Aufstockung der Kapazitäten würden mit großer Wahrscheinlichkeit hohe ökonomische Rendite erzielen, wenn die reale Entwicklung annähernd den dargestellten ehrgeizigen oder moderaten Ausbauszenarien folgt. Überdies sind stimulierende industriepolitische Rahmenbedingungen erforderlich, um die derzeit führende Positionierung deutscher CSP-Unternehmen auf dem internationalen Markt zu behaupten. Anderenfalls droht eine deutliche Reduzierung des möglichen deutschen Wertschöpfungsanteils an CSP-Anlagen.

Die folgenden Punkte könnten wichtige Pfeiler einer effektiven CSP-Förderpolitik sein:

a) Verstärkte Förderung von Forschungs- und Entwicklungsvorhaben mit Schwerpunkt auf Schlüsselkomponenten, für die in besonderem Maße deutsche Expertise vorliegt.

b) Stärkung der Ausbildung junger Fachkräfte mit technischem Know-how zu solarthermischen Kraftwerken.

c) Schaffung von Anreizen für eine Demonstration von CSP-Technologien, um einer Kommerzialisierung der Technologie in großskaligem Maßstab den Weg zu ebnen. 
d) Aufbau bilateraler Partnerschaften oder Dialogforen mit Schlüsselländern zur Förderung der Verbreitung von CSP, insbesondere in Ländern der MENA-Region.

Die hier vorgelegte Analyse der Wertschöpfungschancen deutscher Unternehmen im Segment CSP ist lediglich eine erste große Schätzung. Um präzisere Daten mit einer höheren Aussagekraft zu generieren, sind detaillierte Untersuchungen der deutschen Expertise und Kapazitäten - sowohl Fertigungskapazitäten als auch finanzielle Ressourcen und Human Resources - im Geschäftsfeld CSP vonnöten. Außerdem sollte differenziert werden zwischen der Wertschöpfung deutscher Unternehmen im In- und Ausland. Für diesen Zweck wäre es hilfreich, die Forschungsperspektive von der nationalen Ebene auf andere relevante Weltregionen auszuweiten, um Länder spezifische Rahmenbedingungen (Akteurskonstellationen, Politikrahmen etc.) in die Analyse einbeziehen zu können. Schließlich sollte die Untersuchung nicht auf Parabolrinnenanlagen begrenzt sein, sondern andere CSP-Technologien, insbesondere die Solarturmtechnologie, einschließen.

\section{Quellen:}

ESTELA (2009): Tower Technology, http://www.estelasolar.eu/index.php?id=29, letzter Zugriff am 09.09.2010.

Flabeg (2009): Track Record Solar Mirrors 1983-2010, Nürnberg.

Greenpeace International; EREC (2008): Energy[R]evolution: A Sustainable World Energy Outlook. Amsterdam. http://www.energyblueprint.info/

Greenpeace International; Solar Paces; ESTELA (2009): Global Concentrating Solar Power: Outlook 09. Why Renewable Energy is Hot. Amsterdam.

IEA (2008): Energy Technology Perspectives 2008. Scenarios \& Strategies to 2050, Paris.

Kistner, Rainer (2009): German Participation in the Value-Added Chain of CSP-Plant Construction. Vortrag auf dem Forum "CSP Now!" auf der HannoverMesse am 20. April 2009.

Viebahn P.; Trieb, F., Lechon Y. (2010): The Potential Role of Concentrated Solar Power (CSP) in Africa and Europe - a Dynamic Assessment of Technology Development, Cost Development and Life Cycle Inventories Until 2050. Submitted to Energy Policy, 2010.

Vallentin, D.; Viebahn, P. (2009): Ökonomische Chancen für die deutsche Industrie resultierend aus einer weltweiten Verbreitung von CSP (Concentrated Solar Power)-Technologien. Studie im Auftrag von Greenpeace Deutschland, der Deutschen Gesellschaft Club of Rome und der DESERTEC Foundation. Wuppertal. 